\title{
Online ultrasonic terminal for measuring pig backfat thickness
}

\author{
Ganghong Zhang ${ }^{1,2}$, Wanlin $\mathrm{Gao}^{1,2^{*}}$, Sha Tao ${ }^{1,2}$, Lina $\mathrm{Yu}^{3,4}$, Guofeng Zhang ${ }^{1,2}$, Xuan Luo ${ }^{1,2}$ \\ (1. College of Information and Electrical Engineering, China Agricultural University, Beijing 100083, China; \\ 2. Key Laboratory of Agricultural Informatization Standardization, Ministry of Agriculture, Beijing 100083, China; \\ 3. Institute of Semiconductor, Chinese Academy of Sciences, Beijing, 100083, China; \\ 4. School of Microelectronics, University of Chinese Academy of Sciences, Beijing, 100049, China)
}

\begin{abstract}
The measurement of pig backfat thickness (PBFT) has to stand up to challenges with the reliability, accuracy, and convenience. Acquiring PBFT timely and precisely from a finite distance is extremely necessary to improve the process of pig production and implement effective management. In an attempt to alleviate these problems, an online handheld terminal was designed with a new method based on ultrasonic technology for measuring PBFT during the process of pig breeding, which can overcome the difficulties encountered in other destructive means. The terminal comprised three main components: a main microcontroller unit (MCU) to measure PBFT, a RFID module to identify each pig and send data (e.g. identity, measurement time and PBFT) to a server via wireless transmission module, and an ultrasonic transducer to drive and receive signals between them. A measurement error within 0-1 mm was acquired through testing three groups of samples. Results indicated that this handheld terminal had a required accuracy and proved that the ultrasonic wave processing method can be deployed in a mobile terminal for PBFT measurement. It also provided a feasible nondestructive alternative to measure PBFT. Associated with information management software platform, this method may ultimately help pig production farmers measure the PBFT accurately and conveniently, and improve the pig production efficiency.
\end{abstract}

Keywords: pig backfat thickness, nondestructive measurement, ultrasonic technology, online terminal, pig production DOI: $10.25165 / \mathrm{j}$.jjabe.20181102.3278

Citation: Zhang G H, Gao W L, Tao S, Yu L N, Zhang G F, Luo X. Online ultrasonic terminal for measuring pig backfat thickness. Int J Agric \& Biol Eng, 2018; 11(2): 190-195.

\section{Introduction}

Pig industry, as a traditional industry in China, plays a crucial role in economic development ${ }^{[1]}$. Pork, as an indispensable part of agricultural and sideline products for daily life, occupies a dominant position in meat products in China. Pig backfat thickness (PBFT) is a crucial predictor of carcass lean content and meat quality, and as the economic performance, reveals one of the most important breeding targets in pig improvement ${ }^{[2-9]}$. The PBFT also reflects the growth rate, pork percentage and it has a paramount impact on the reproductive system and also reflects lean meat percentage, and as an important indicator also used to evaluate pork quality grade ${ }^{[4]}$. Accordingly, rapidly acquiring the backfat thickness becomes an effective method to get the growth rate of a pig. Dynamically acquiring PBFT in the growth stage will provide benefits on the breeders to control the feeding, and fairly safely contribute to manage the overall process for smart farming. Traditionally, methods of getting the PBFT data by operating with knife, probe and ruler ${ }^{[10]}$ exist some disadvantages

\section{Received date: 2017-02-24 Accepted date: 2018-01-22}

Biographies: Ganghong Zhang, PhD candidate, research interests: agricultural application specific chip and deep learning, Email: kevinfoster@163.com; Sha Tao, PhD, research interests: agricultural products inspection, Email: taosha20070608@163.com; Lina Yu, PhD, research interests: agricultural products inspection, Email: linda-3740@163.com; Guofeng Zhang, PhD candidate, research interests: agricultural application specific chip, Email: gfky2010@sina.com; Xuan Luo, Master, research interests: agricultural big data, Email:285879095@qq.com.

*Corresponding author: Wanlin Gao, PhD, Professor, research interests: agricultural informatization and agricultural application specific chip. College of Information and Electrical Engineering, China Agricultural University, Beijing 100083, China. Tel: +86-10-62736755, Email: wanlin_cau@163.com. in this process because of the pork surface easily contaminated by direct touching. Furthermore, the detection speed and data storage also become more and more important.

Ultrasonic technology has been widely applied in animal science ${ }^{[11-19]}$, and can play a crucial role in addressing the above mentioned issues, and this experiment presents an online terminal technique that is based on ultrasonic technology for measuring the PBFT with a quick, secure and non-destructive method which also provides real time storage and transmission.

\section{Materials and methods}

\subsection{Principle for detecting PBFT}

During the process of measurement, using the ultrasonic pulse echo method ${ }^{[20]}$ to measure the backfat thickness and the method of pulse reflection technique for thickness measurement will apply ultrasonic probe laser and pulse wave into the subject, and determine the material thickness according to the reflection wave of observed object in different materials. Superficial tissue of a pig mainly consists of dermal, fat, muscle and protein etc. The acoustic impedance becomes different because of different tissue components. Therefore, the ultrasonic wave propagation in pig tissue encountering the interface of the two kinds of different organizations will produce a changed the optical phenomena such as reflection, refraction. Through the reflection getting distance of ultrasonic propagation, the PBFT can be obtained. Table 1 show the values of sound velocity, density and acoustic impedance ${ }^{[21]}$ regarding the different superficial soft tissues, which can be used as the experimental samples of acoustic characteristic value as the referred standard.

The process of thickness measurement is as follows: firstly an ultrasonic transducer emits ultrasound, and it will generate 
ultrasonic echo when ultrasound meets obstacles in the process of transmission. Then ultrasonic transducer receives the echo signal and computes the time between launching ultrasonic waves and receiving echo signal. Consequently, the distance between transducer and obstacle can be calculated through Equation (1). Diagram of the pulse reflection is shown in Figure 1.

Table 1 Values of sound velocity and acoustic impedance in superficial tissue

\begin{tabular}{cccc}
\hline Tissue & $\begin{array}{c}\text { Density } \rho \\
/ \mathrm{g} \cdot \mathrm{cm}^{-3}\end{array}$ & $\begin{array}{c}\text { Sound velocity } \\
/ \mathrm{cm} \cdot \mathrm{s}^{-1}\end{array}$ & $\begin{array}{c}\text { Acoustic impedance } \\
/ \mathrm{Pa} \cdot \mathrm{s} \cdot \mathrm{m}^{-1}\end{array}$ \\
\hline Fat & 0.592 & 1450 & $1.38 \times 10^{6}$ \\
Muscle & 1.08 & 1580 & $1.70 \times 10^{6}$ \\
Soft tissue & 1.06 & 1540 & $1.60 \times 10^{6}$ \\
\hline
\end{tabular}

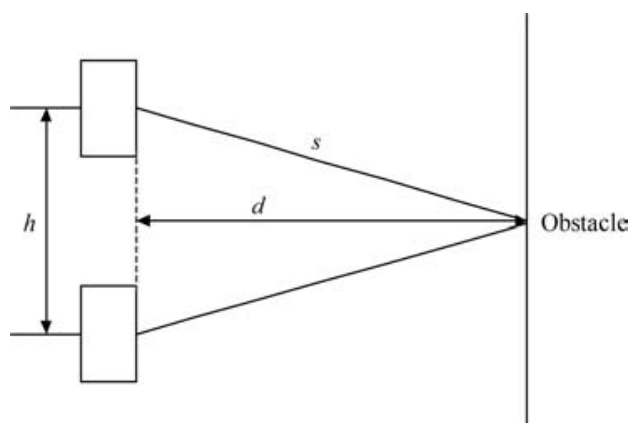

Figure 1 Diagram of the pulse reflection

Calculation equation is:

$$
d=v t / 2
$$

where, $d$ is the distance between transducer and obstacle, $\mathrm{m} ; t$ represents the time between launching ultrasonic wave and receiving echo signal, $\mathrm{s} ; \quad v$ represents ultrasonic propagation velocity in the current medium, $\mathrm{m} / \mathrm{s}$.

This method has the advantages of simple operation, high sensitivity, and accurate positioning, and can generate an intensely high resolution for the minimal discontinuous interface and accurately measure the discontinuous depth at the bottom of the interface.

\subsection{System structure}

The main modules of measurement system for PBFT include data collection module, RFID module, data processing module and wireless transmission module. The system framework is shown in Figure 2.

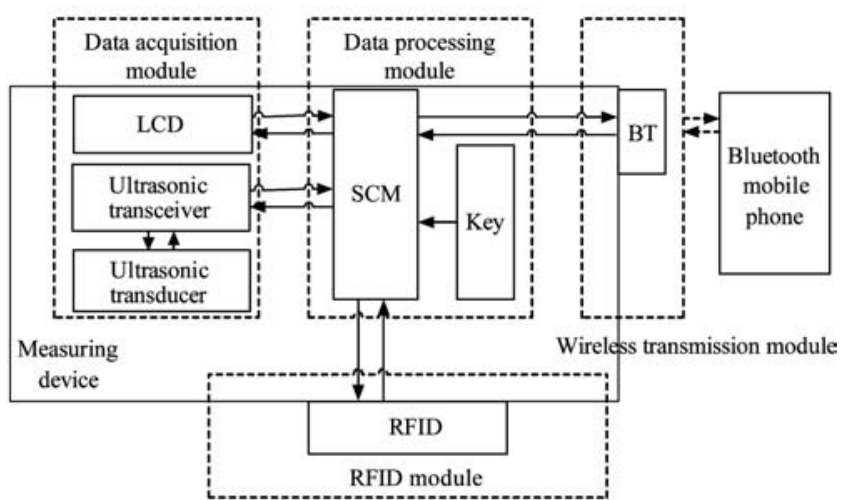

Figure 2 Diagram of the system framework

The data acquisition module used to collect data for calculating through ultrasonic technology designed to detect data. With this method, no need to destructively obtain pigs' vivo information. And ultrasonic wave has the features of high longitudinal resolution and strong anti-interference ability, and it is easy to realize miniaturization and integration. Subsequently, the measured information is sent to the data processing module for analysis, calculation and storage. When the measured data in the data processing module got the relevant processing, and can directly send the information to a smart phone via wireless transmission module. Moreover, before measurement action, firstly identify each pig by means of RFID which obtains individual information concerning each pig in order to trace the related record. Bluetooth technology is experimented for data transmission in this experiment, and widely used transfer function in mobile phone at present time.

(1) Data acquisition module

The module is divided into three parts: transmission, receiving and ultrasonic transducer. Ultrasonic transducer is used to implement the conversion of sound energy and mechanical energy. Transmission and receiving modules are used for signal transmission, filtering, amplifying and shaping.

(2) Data processing module

The data processing module is used to analyze, calculate and store the signal measured by the data acquisition module, and controls the whole hardware system, mainly including microprocessor, keyboard, display, buzzer, external memory and quite a few external interfaces like Bluetooth and so on.

(3) The RFID module

RFID module is used to record authentication, tracking and growth information of different pigs.

(4) Wireless transmission module

Wireless transmission module used to realize the interaction between the measurement device and the intelligent mobile phone for sending the data to the mobile phone.

\subsection{Main control chip}

The STM32F103R6 ${ }^{[22]}$ enhanced by a single MCU chip for measuring the PBFT in this present research, its main functions are as follows: (1) Controlling ultrasonic transmitter circuit for transmitting and receiving ultrasonic signals; (2) Calculating the ultrasonic propagation time in superficial fat; (3) Calculating the thickness according to the ultrasonic spreading time in superficial fat; (4) Displaying the PBFT by the display module; (5) Controlling the RFID to read identification information from a pig's ear tag; (6) Sending all the information to a data server through the wireless module.

Enhanced chip STM32F103R6 using high-performance 32-bit ARM Cortex-M3 core based on the reduced instruction set computer (RISC) architecture; its operating frequency is $72 \mathrm{MHz}$ and working voltage is 2-3.6 V. Built-in $64 \mathrm{k}$ bytes flash and $20 \mathrm{k}$ bytes static random access memory (SRAM). Even possess rich enhanced input/output (I/O) port and two advanced peripheral bus (APB). What's more, it has two analog-to-digital(A/D) converters, three general 16-bit timers and a pulse width modulation (PWM) timer; owns standard and advanced interaction interface: two inter-integrated circuit $\left(\mathrm{I}^{2} \mathrm{C}\right)$, three serial peripheral interfaces (SPI), five universal synchronous/asynchronous receiver/transmitter (USART), one universal serial bus (USB) port and one controller area network (CAN) bus. These features make performance line microcontroller family suitable for a wide range of applications. It has been developed to provide a low-cost platform that meets the needs of MCU implementation, with a reduced pin count and lowowner consumption, while delivering outstanding computational performance and advanced system response to interrupts.

\subsection{Ultrasonic transmission circuit}

The ultrasonic transmission circuit consists of two parts: 
ultrasonic transducer and ultrasonic excitation circuit. The higher center frequency of the transducer can obtain better response. But the measuring distance is shorter, and at the same time the price is higher and needs higher excitation voltage, usually almost dozens or even hundreds of volts. Considering the PBFT is usually between $5-40 \mathrm{~mm}$ and the cost of hardware, a one-piece probe transceiver with center frequency of $1.5 \mathrm{MHz}$ was selected in this paper. The ultrasonic excitation circuit works when instantaneously loading a certain form of voltage signal at both ends of the transducer and the transducer will convert the electrical signals into acoustic signals and emit.

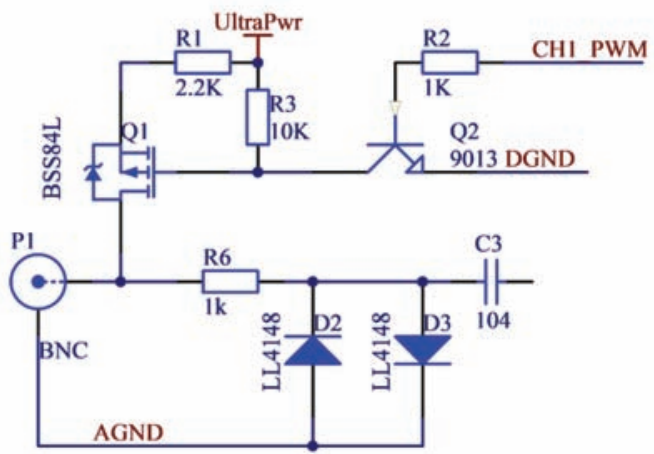

Figure 3 The ultrasonic transmission circuit

The ultrasonic transmission circuit is as shown in Figure 3. The working principle of ultrasonic transmission circuit: when the system starts working, the MCU gives high voltage level through the pin of CH1_PMW opening the Q2 and then opening the Q1. At this time, the transducer driving power (UltraPwr) with capacity of $30 \mathrm{v}$ consuming time almost $200 \mathrm{~ns}$. It will produce an excitation signal with high-pressure negative pulse and the ultrasonic transducer will generate mechanical movement to transmit ultrasonic because the ultrasonic transducer is a sensing component, the system does not require a special signal generating circuit because it itself could transmit ultrasonic with the self-oscillating. Through this method, it reduces the signal interference of the transmission circuit to the receiving circuit even reduces the power consumption of the system and saves the cost of hardware.

\subsection{Ultrasonic echo-receiving circuit}

Echo receiving circuit used to detect the reflected signal when the launched ultrasonic meets different mediums, is divided into signal collecting and signal modulation circuit. There is a certain attenuation degree when the ultrasonic propagating in the medium and the attenuation degree will be proportional with the propagation distance. Thus the received acoustic signals become faint. At the same time, the received signals by the ultrasonic transducer play a role of pulse signal in nature before transferring for further processing, therefore the interference and noise will be removed firstly after removing foreign substances then transferred to a single chip microcomputer and microcontroller. Extracting the effective signals and magnifying them to increase the signal-to-noise ratio. Finally, effective signals are analyzed by the single chip microcomputer (SCM). Ultrasonic echo receiving circuit is as shown in Figure 4.

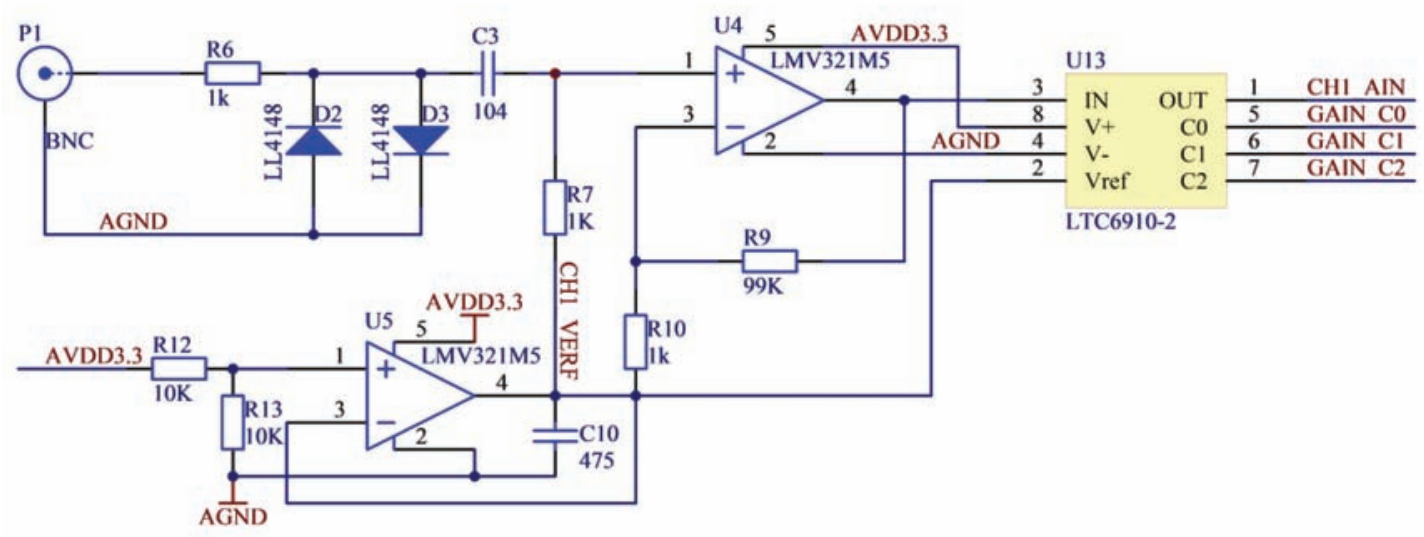

Figure 4 Ultrasonic echo-receiving circuit

There may be a multitude of interference signals exist in the received signals detected by the ultrasonic transducer, like the cross interference between sensors, power line interference, etc. Because of the interference signal concentrated in low frequency, the band-pass filter circuit used to filter out the effective signals becomes feasible. As shown in Figure 4, C3 and R7 form a high pass filter, frequency of $0.16 \mathrm{MHz}$ used to filter the waves below $0.16 \mathrm{MHz}$. The ADC of the microchip as a single supply cannot detect the negative voltage; the AC signals should be converted to $0-3.3 \mathrm{~V}$ and the bias voltage with $1.65 \mathrm{~V}$.

The device adopts the LMV321M5 operational amplifier with dual operational amplifier to amplify the signals. The operational amplifier has a high power bandwidth and small-signal bandwidth, the noise performance becomes better than an army of standard operational amplifiers with lower noise. In Figure 4, the amplification factor is: $(\mathrm{R} 9+\mathrm{R} 10) / \mathrm{R} 10=100$.

\subsection{Transmission module}

In the system, the transmission module used the Bluetooth technology based around the BT-HC07 which produced by
Guangzhou Huicheng Information Technology Co., Ltd. It uses the type of flash named CSR BC4+8M FLASH and has the pins of PIO0-PIO11, AIO0, AIO1, USB, PCM, UART and SPI. There is an $8 \mathrm{M}$ FLASH in it. With such a powerful function, users can customize the software. As a slaver, it is suitable for a variety of Bluetooth devices built-in RF antenna, so it is easy to debug. The Bluetooth module is compliant with Bluetooth Specification of $2.0+$ EDR, which is the first one that supports for enhanced transfer rate specification of Bluetooth solutions ${ }^{[23]}$. The Bluetooth module circuit is as shown in Figure 5.

\subsection{RFID module}

RFID directly selected in this paper has been well integrated with MF522-AN, which adopted the Philips original chip-MFRC522 designed to the card reader circuit. MF522-AN module has low power dissipation, low cost, and small volume, is a highly integrated reader/writer IC for contactless communication. The module uses a supply voltage of $3.3 \mathrm{~V}$, and interacts with the system CPU via the $\mathrm{I}^{2} \mathrm{C}$ interface. It has efficient and reliable stability and can read card at a certain distance. Selected 
contactless smart card named S50 card as an electronic tag that produced by NXP MF1 IC S50 Company.

The circuit of RFID module is as shown in Figure 6. This module communicates with the controller using $\mathrm{I}^{2} \mathrm{C}$ interface. Pin 4 and Pin 5 are respectively linked with the Pin SCL and Pin SDA of the SCM to read and write, and controlling CMOS to start/stop the whole module to work through Pin RFID_EN.

U3

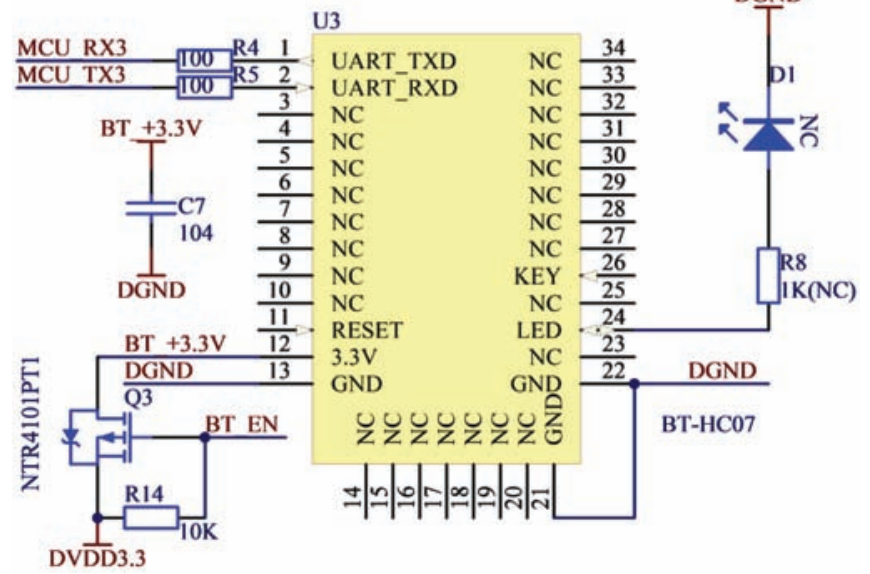

Figure 5 Circuit of Bluetooth wireless transmissions

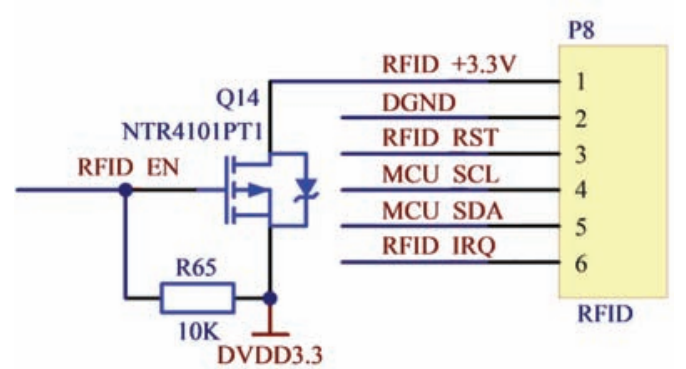

Figure 6 Circuit of RFID module

\subsection{Pig backfat thickness measurement}

When pressing the 'measurement' button on the keyboard, the SCM will start measuring the backfat thickness, and the transducer will produce oscillation signals and translate them into ultrasonic signal to launch out. And then the data acquisition module begins to work as soon as transducer receives the echo signals pushed into the CPU to calculate. The flow chart of the PBFT measurement is shown in Figure 7.

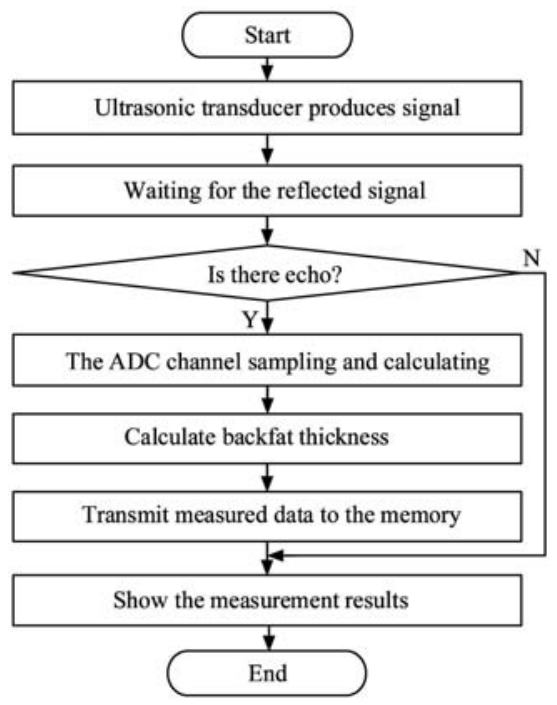

Figure 7 Flowchart of backfat thickness measurement
According to the working principle of ultrasonic pulse reflection, the calculation equation of backfat thickness is as follows:

$$
S=1500 \Delta t \times 10^{-3} / 2
$$

where, $\mathrm{S}$ represents PBFT and its unit is millimeter, $\mathrm{mm} ; \Delta t$ is the total passing time and its unit is microsecond, $\mu \mathrm{s}$.

The size of backfat thickness is changing with $\Delta t$ which represents the passing time. The propagation rate of ultrasonic in the fat and soft tissue is $1450 \mathrm{~m} / \mathrm{s}$ and $1540 \mathrm{~m} / \mathrm{s}$ respectively. In order to find a suitable velocity parameter, by operating an ultrasonic reference block (thickness $40 \mathrm{~mm}$ ) which the organization structure is the same with a pig's fat layer, and consequently, the velocity parameter with $1500 \mathrm{~m} / \mathrm{s}$ is selected.

\subsection{Data storage format}

The PBFT collected by the SCM needs to be stored as a history data and can be formatted as: tag number, operation time (year, month and date) and thickness. The data storage format is as shown in Figure 8. The operation time takes three bytes, and both tag number and thickness one byte. Only five bytes is used for the storage and $1 \mathrm{Mb} \quad \mathrm{I}^{2} \mathrm{C}$-compatible 2-wire Serial EEPROMAT24CM01 can be optional in this paper.

\begin{tabular}{|ccc|}
\hline A0 A1 A2 & B0 & C0 \\
\hline time (3 bytes) & tag number (1 byte) & thickness (1 byte)
\end{tabular}

Figure 8 Data storage format

\subsection{Samples selection and test}

Two types of samples are used to check the prototype device. One is fresh superficial tissue from the pig back got within one hour after slaughtering, and the number of samples is 10 , that are from a different pig positon. And cut the tissue into square, which takes a pig's skin as the bottom with the area of $20 \mathrm{~cm}$ by $20 \mathrm{~cm}$ and avoid being pressed. Use a pen to make marks on the skin of each tissue sample to ensure protection. Another sample is transparent plastic test specimen (thickness $25 \mathrm{~mm}$ ) which used to adjust ultrasonic measuring instrument, and its tissue structure is same as the fat. Figure 9 is the picture of the terminal for PBFT. Each sample was measured 10 times and averaged.

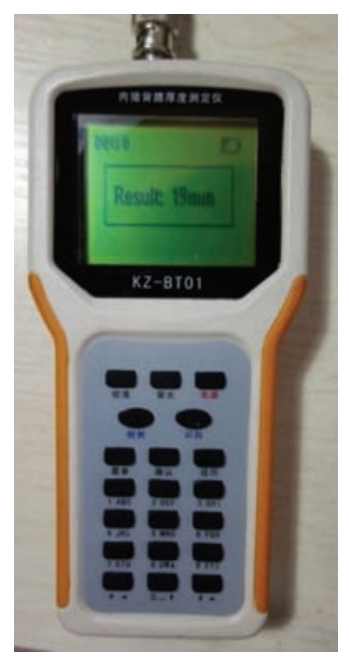

Figure 9 Photo of the ultrasonic measurement terminal

\section{Results and discussion}

Results of an experimental application of this terminal analysis procedure are given to illustrate the proposed technique. Typical measurement results are shown in Table 2 . 
Table 2 Measurement results with prototype device/ruler (mm)

\begin{tabular}{cccc}
\hline No. & Sample 1 & Sample 2 & Sample 3 \\
\hline 1 & $18.00 / 19.00$ & $26.00 / 27.00$ & $28.00 / 29.00$ \\
2 & $18.00 / 19.00$ & $26.00 / 27.00$ & $28.00 / 29.00$ \\
3 & $18.00 / 19.00$ & $26.00 / 27.00$ & $28.00 / 29.00$ \\
4 & $17.00 / 18.50$ & $25.00 / 26.50$ & $27.00 / 28.00$ \\
5 & $17.00 / 18.50$ & $25.00 / 26.50$ & $27.00 / 28.00$ \\
6 & $17.00 / 18.00$ & $25.00 / 26.50$ & $27.00 / 28.00$ \\
7 & $17.00 / 18.00$ & $25.00 / 26.00$ & $27.00 / 28.00$ \\
8 & $16.00 / 16.50$ & $25.00 / 26.00$ & $26.00 / 27.00$ \\
9 & $16.00 / 16.00$ & $25.00 / 25.00$ & $26.00 / 26.00$ \\
10 & $16.00 / 16.00$ & $25.00 / 25.00$ & $26.00 / 26.00$ \\
Average & $17.00 / 17.85$ & $25.30 / 26.25$ & $27.00 / 27.80$ \\
\hline
\end{tabular}

The corresponding result correlation analysis is shown in Figure 10.
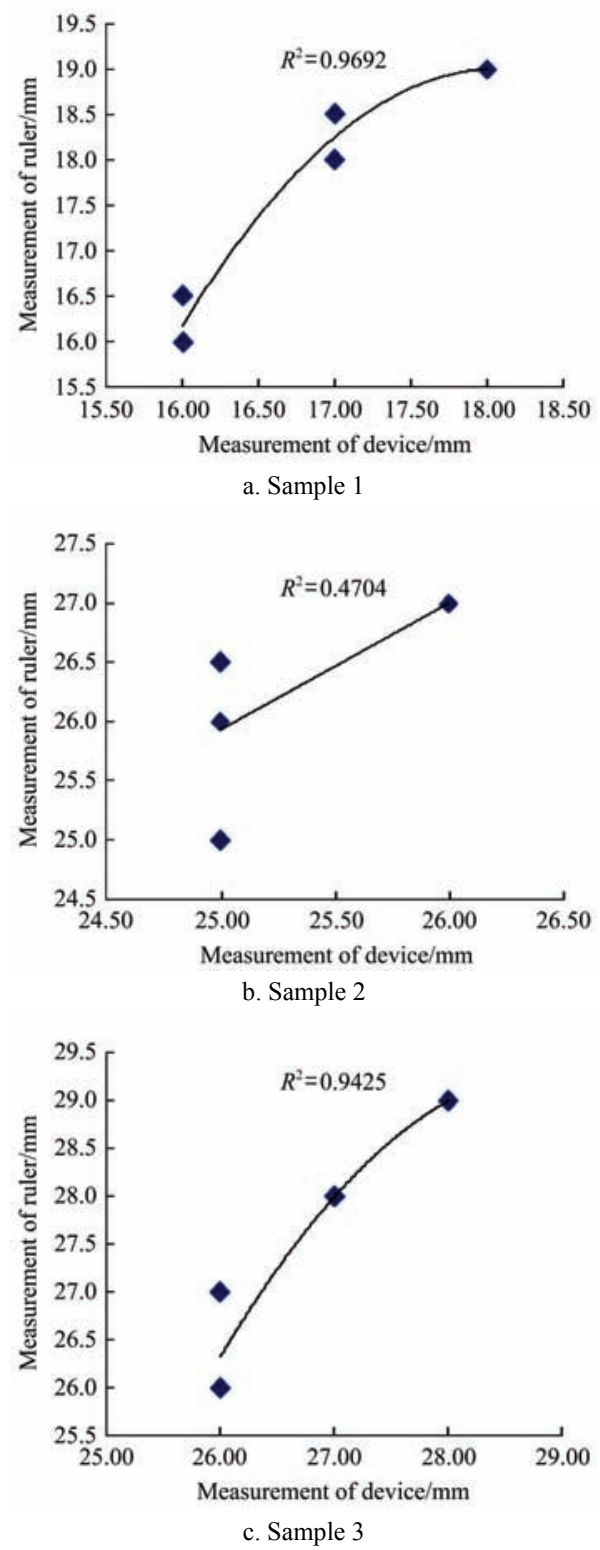

Figure 10 Diagram of result correlation analysis

From the measurement results of samples 1-3 in Table 2 and Figures 10a-10c, we can observe that the measurement results are almost consistent when using both the device and the ruler from the three different samples. Although there is no error when testing the known standard specimen using both the device and the ruler, there exists a measurement error within $0-1 \mathrm{~mm}$, compared with Renco Lean Meter, measurement error with $\pm 1 \mathrm{~mm}$ (produced by RENCO ELECTRONICS, INC.), the terminal presented can be implemented more easily, with similar efficiency and accuracy results. Possibly existed error produced by human mistakes during the measurement process or maybe the parameter of ultrasonic transmission rate chosen was inadequate or maybe the test objects possess some issue. Overall, as it could be seen the experimental data, the measurement results demonstrated a certain reliability, not stable, but feasible to improve a high practical value when using the device.

\section{Conclusions}

Based on the ultrasonic measuring technology, an online, interactive, and portable terminal for measuring pig backfat thickness was developed by combining MCU module, RFID module and an ultrasonic transducer. It can be applied to the process of pig production and improve the efficiency when using contactless or nondestructive method. The measurement error was within $0-1 \mathrm{~mm}$. The test results indicated that this handheld device had the characteristics of high precision, low power consumption, easy to carry, easy to operate and low cost comparing with the similar devices. Associated with information management software platform, this method may ultimately help pig production farmers measure the PBFT accurately and conveniently, and improve the pig production efficiency.

\section{Acknowledgements}

This work was financially supported by Project of Science Operating Expenses from Ministry of Education of China (Grant No. 2017PT19) and Chinese Universities Scientific Fund (Grant No. 2017XD003).

\section{[References]}

[1] Baldassini W A, Chardulo L A L, Silva J A V, Malheiros J M, Dias V A D, Espigolan R, et al. Meat quality traits of Nellore bulls according to different degrees of backfat thickness: a multivariate approach. Animal Production Science, 2016; 57(2): 363-370.

[2] Jong J A D, Derouchey J M, Tokach M D, Goodband R D, Dritz S S, Nelssen J L, et al. Effects of corn particle size, complete diet grinding, and diet form on finishing pig growth performance, caloric efficiency, carcass characteristics, and economics. Kansas State University Swine Day 2012. Report of progress 1074, 2012-10; pp.316-324.

[3] Zambonelli P, Gaffo E, Zappaterra M, Bortoluzzi S, Davoli R. Transcriptional profiling of subcutaneous adipose tissue in Italian large white pigs divergent for backfat thickness. Animal Genetics, 2016; 47(3): 306-323.

[4] Grzes M, Sadkowski S, Rzewuska K, Szydlowski M, Switonski M. Pig fatness in relation to fasn and insig2 genes polymorphism and their transcript level. Molecular Biology Reports, 2016; 43(5): 381-389.

[5] Egea M, Linares M B, Garrido M D, Madrid J, Hernández F. Feeding Iberian duroc cross pigs with crude glycerine: effects of diet and gender on carcass and meat quality. Meat Science, 2016; 111: 78-84.

[6] Roongsitthichai A, ummaruk P. Importance of backfat thickness to reproductive performance in female pigs. Thai Veterinary Medicine, 2014; 44(2): 171-178.

[7] Schwarz T, Turek A, Nowicki J, Tuz R, Rudzki B, Bartlewski P M. Production value and cost-effectiveness of pig fattening using liquid feeding or enzyme-supplemented dry mixes containing rye grain. Czech J. Anim. Sci., 2016; 61(8): 341-350.

[8] Biermann A D, Yin T, Uu K V B, Rübesam K, Kuhn B, König S. From phenotyping towards breeding strategies: using in vivo indicator traits and genetic markers to improve meat quality in an endangered pig breed. Animal, 2015; 9(6): 919-927. 
[9] Knecht D, Duziński K, Lisiak D. Accuracy of estimating the technological and economic value of pig car. Canadian Journal of Animal Science, 2016; 96(1): 37-44.

[10] Huang Y C, Hong-Jun L I, Qin, G, Wang T. Effect of processing methods and time on intramuscular lipid content and fatty acid composition of pork. Science \& Technology of Food Industry, 2012; 33(1): 159-158.

[11] Agamy R, Abdel-Moneim A Y, Abd-Alla M S, Abdel-Mageed I I, Ashmawi G M. Use of ultrasound measurements to predict carcass characteristics of Egyptian ram-lambs. Asian Journal of Animal \& Veterinary Advances, 2008; 10(5): 203-214.

[12] Miar Y, Plastow G S, Bruce H L, Moore S S, Durunna O N, Nkrumah J D, et al. Estimation of genetic and phenotypic parameters for ultrasound and carcass merit traits in crossbred beef cattle. Canadian Journal of Animal Science, 2014; 94(2): 273-280.

[13] Kennedy N, Quinton A E, Martin A, Peek M J, Nanan R. Ultrasound measurement of subcutaneous fat thickness as an independent predictor for adverse pregnancy outcomes. Ultrasound in Obstetrics \& Gynecology, 2015; 44(S1): 321-321

[14] Kopinski S, Engel T, Cassel M, Fröhlich K, Mayer F, Carlsohn A. Ultrasound applied to subcutaneous fat tissue measurements in international elite canoeists. International Journal of Sports Medicine, 2015; 36(14): 1134-1141

[15] Peña F, Molina A, Juárez M, Requena F, Avilés C, Santos R, et al. Use of serial ultrasound measures in the study of growth- and breed-related changes of ultrasonic measurements and relationship with carcass measurements in lean cattle breeds. Meat Science, 2014; 96(1): 247-55.

[16] Corona E, García-Pérez J V, Santacatalina J V, Ventanas S, Benedito J. Ultrasonic characterization of pork fat crystallization during cold storage. Journal of Food Science, 2014; 79(5): E828-E838.

[17] Kachanov V K, Sokolov I V, Kontsov R V, Sinitsyn A A, Fedorov M B. Adaptive instruments for ultrasonic nondestructive testing of large objects with complex structures. Russian Journal of Nondestructive Testing, 2016 52(5): 251-260.

[18] Awad T S, Moharram H A, Shaltout O E, Asker D, Youssef M M. Applications of ultrasound in analysis, processing and quality control of food: A review. Food Research International, 2012; 48(2): 410-427.

[19] Pingret D, Fabiano-Tixier A S, Chemat F. Degradation during application of ultrasound in food processing: A review. Food Control, 2013; 31(2): 593-606.

[20] Jin Y Y, Gao W L, Zhang H, An D, Guo S H, Ahmed S I, Liu Y L. Identification of damaged corn seeds using air-coupled ultrasound. Int J Agric \& Biol Eng, 2016; 9(1): 63-70.

[21] Mamou J, Tamura K., Rohrbach D, Yamaguchi T, Franceschini E. Relationship between ultrasound scattering and acoustic impedance maps in sparse and dense random media. Journal of the Acoustical Society of America, 2017; 142(4): 2536-2536.

[22] ARM-based 32-bit MCU STM32F101xx and STM32F103xx firmware library User manual, STMicroelectronics, 2008.

[23] Ferro E, Potorti F. Bluetooth and Wi-Fi wireless protocols: a survey and a comparison. IEEE Wireless Communications, 2005; 12(1): 12-26. 